\title{
Sobrevida e perfis social, clínico e hemodinâmico de pacientes admitidos em Unidade de Terapia Intensiva que evoluíram a óbito
}

\author{
Survival and social, clinical and hemodynamic profiles of patients admitted to the Intensive \\ Care Unit who died
}

\section{Perfiles de supervivencia y social, clínico y hemodinámico de pacientes ingresados en la Unidad de Cuidados Intensivos que fallecieron}

Giovana Salomão Melo ${ }^{1 *}$, Edila Monteiro de Andrade ${ }^{1}$, Thalia Saraiva Mendonça ${ }^{1}$, Isabella Boechat Faria Santos ${ }^{1}$, Cleuma Oliveira Soares ${ }^{1}$, Fabiana de Campos Gomes ${ }^{2}$, João Simão de Melo Neto ${ }^{1}$.

\section{RESUMO}

Objetivo: Caracterizar os perfis social, clínico e hemodinâmico de pacientes admitidos em Unidade de Terapia Intensiva (UTI) que evoluíram a óbito e verificar a influência na sobrevida. Métodos: Coleta de prontuários de pacientes admitidos na UTI de um Hospital de referência terciária na região amazônica, que evoluíram a óbito $(n=200)$, com análise das variáveis clínicas, sociais e hemodinâmicas e descrição das frequências absoluta e relativa, média, desvio padrão e intervalo de confiança de $95 \%$. Resultados: Características sociais predominantes: idade $20-59$ anos $(48,5 \%)$, sexo masculino (52\%), raça/cor pardo (72,5\%), profissão empregada doméstica (10,5\%), religião católica (6,5\%), escolaridade 9 anos (21\%) e estado civil solteiro (30\%). Características clínicas principais: doenças respiratórias (20,5\%), estado grave na admissão (70,5\%), coma induzido (42\%), acamados (87\%), uso de ventilação mecânica invasiva (91\%) e média de aspiração brônquica 1,27 $\pm 0,55$ por dia. Complicações clínicas prevalentes: sepse $(79,5 \%)$, choque $(69 \%)$ e edema $(61 \%)$. Uso de sonda vesical $(82,5 \%)$ e tempo médio de internação $11,81 \pm 9,50$ dias. As características hemodinâmicas acentuadas foram: creatinina, uréia, lactato e leucócitos. Além disso, observou-se menor sobrevida de indivíduos da raça Branca e com leucocitose admissional. Conclusão: Este estudo auxilia na compreensão do perfil epidemiológico da população local, contribuindo no melhor manejo do paciente crítico.

Palavras-chave: Unidade de Terapia Intensiva, Perfil de saúde, Monitorização hemodinâmica, Sobrevida, Óbito.

\section{ABSTRACT}

Objective: To characterize the social, clinical, and hemodynamic profiles of patients admitted to the Intensive Care Unit (ICU) who died and to verify the influence on survival. Methods: Collection of medical records of patients admitted to the ICU of a tertiary referral hospital in the Amazon region, who died $(n=200)$, with analysis of clinical, social and hemodynamic variables and description of absolute and relative frequencies, mean, standard deviation and interval $95 \%$ confidence. Results: Predominant social characteristics: age 20-59 years (48.5\%), male (52\%), race / brown color $(72.5 \%)$, profession as a domestic worker $(10.5 \%)$, Catholic religion $(6,5 \%), 9$ years of schooling $(21 \%)$ and single marital status $(30 \%)$. Main clinical characteristics: respiratory diseases $(20.5 \%)$, severe condition on admission $(70.5 \%)$, induced coma $(42 \%)$, bedridden $(87 \%)$, use of invasive mechanical ventilation $(91 \%)$ and average aspiration bronchial $1.27 \pm 0.55$ per day. Prevalent clinical complications: sepsis $(79.5 \%)$, shock $(69 \%)$ and edema $(61 \%)$. Use of a bladder catheter $(82.5 \%)$ and mean hospital stay $11.81 \pm 9.50$ days. The accentuated hemodynamic characteristics were: creatinine, urea, lactate, and leukocytes. In addition, less survival was observed among individuals of the White race and with admission leukocytosis. Conclusion: This study helps to understand the epidemiological profile of the local population, contributing to better management of critical patients.

Keywords: Intensive Care Unit, Health profile, Hemodynamic monitoring, Survival, Death.

\section{RESUMEN}

Objetivo: caracterizar los perfiles sociales, clínicos y hemodinámicos de pacientes ingresados en la Unidad de Cuidados Intensivos ( $\mathrm{UCl}$ ) que fallecieron y verificar la influencia en la supervivencia. Métodos: Colección de registros médicos de pacientes ingresados en la UCI de un hospital terciario de referencia de la Amazonía, fallecidos $(n=200)$, con análisis de variables clínicas, sociales y hemodinámicas y descripción de frecuencias absolutas y relativas, media, desviación estándar e intervalo. 95\% de confianza. Resultados: características sociales predominantes: edad 20-59 años (48.5\%), hombre (52\%), raza / color marrón $(72.5 \%)$, profesión como trabajadora doméstica (10.5\%), religión católica $(6.5 \%), 9$ años de escolaridad $(21 \%)$ y estado civil soltero (30\%). Principales características clínicas: enfermedades respiratorias $(20.5 \%)$, afección grave al ingreso $(70.5 \%)$, coma inducido (42\%), postrado en cama (87\%), uso de ventilación mecánica invasiva (91\%) y aspiración promedio bronquial $1.27 \pm 0.55$ por día. Complicaciones clínicas frecuentes: sepsis $(79.5 \%)$, shock $(69 \%)$ y edema $(61 \%)$. Uso de una sonda vesical $(82,5 \%)$ y estancia hospitalaria media $11,81 \pm 9,50$ días. Las características hemodinámicas acentuadas fueron: creatinina, urea, lactato y leucocitos. Además, se observó una menor supervivencia en individuos de la raza blanca y con leucocitosis de admisión. Conclusión: este estudio ayuda a comprender el perfil epidemiológico de la población local, contribuyendo a un mejor manejo de los pacientes críticos.

Palabras clave: Unidades de Cuidados Intensivos, Perfil de salud, Monitorización hemodinámica, Sobrevida, Muerte.

\footnotetext{
1Universidade Federal do Pará (UFPA), Belém - PA. *E-mail: giovana.salomao@gmail.com
}

${ }^{2}$ Faculdade de Medicina de São José do Rio Preto (FAMERP), São José do Rio Preto - SP. 


\section{INTRODUÇÃO}

A Unidade de Terapia Intensiva (UTI) é um local destinado ao cuidado progressivo dos pacientes em estado grave ou crítico, seja no âmbito clínico ou cirúrgico, os quais passaram por avaliações periódicas que indicaram a necessidade de acolhimento e de cuidados intensivos. O amparo do paciente é contínuo e assegurado por uma equipe multidisciplinar e especializada de médicos, enfermeiros e fisioterapeutas que são qualificados a monitorar e exercer o uso de equipamentos e executar procedimentos, visando a segurança e a resolubilidade das demandas e alta hospitalar. Esse cuidado progressivo deve ser baseado nas complicações e condições clínicas referentes ao que for recomendado ao paciente (MINISTÉRIO DA SAÚDE, 2017).

A admissão do paciente se dá por meio de vários fatores, como doença pulmonar vinculada à necessidade de ventilação mecânica invasiva ou não, com risco de falência respiratória, bloqueio de vias aéreas e necessidade de suplementação de oxigênio. Além disso, outros exemplos de enfermidades que corroboram para a necessidade de cuidados intensivos são aquelas relacionadas ao sistema cardiovascular, como insuficiência cardíaca (necessitando de suporte hemodinâmico e acompanhamento da pressão arterial) e ao sistema neurológico, acompanhadas de riscos convulsivos, broncoaspiração e traumatismo cranioencefálico (MINISTÉRIO DA SAÚDE, 2017).

A internação em uma UTI objetiva reduzir a morbimortalidade associada a doenças críticas. Contudo, apesar dos avanços tecnológicos, a evolução ao óbito ainda é frequente e as taxas de mortalidade são influenciadas por diversas condições (COOK D e ROCKER G, 2014).

Os aspectos sociodemográficos podem interferir no tempo de internação hospitalar e, consequentemente, no desfecho (ABE T, et al., 2018). Os países desenvolvidos e em desenvolvimento apresentam realidades distintas quanto à idade, gênero, etnia e comorbidade de pacientes admitidos em UTI (MELO FAF, et al., 2020). O perfil dos pacientes é capaz de refletir a realidade de cada região e permite o estudo da propagação e frequência das doenças, seu modo de distribuição e sua evolução, possibilitando melhor manejo de pacientes críticos.

A monitorização hemodinâmica - invasiva ou não invasiva - está diretamente ligada à evolução do paciente crítico, uma vez que, ao analisar esses parâmetros é possível acompanhar situações de instabilidade hemodinâmica como choques e má perfusão ou hipóxia tecidual. Do mesmo modo, é viável acompanhar a situação gasométrica, volêmica e de outros parâmetros, para que a equipe intensivista possa definir uma terapia e verificar a resposta desta terapia e seus possíveis desfechos (CANO AG, et al., 2012).

Além disso, o monitoramento de variáveis hemodinâmicas é vital para melhorar as chances de sobrevida do paciente, servindo de parâmetro à resposta ao tratamento e à gravidade do estado de saúde (XIA DL, et al., 2016). Levando em consideração esse cenário, traçar um perfil social, clínico e hemodinâmico se torna de grande importância para a melhor compreensão sobre os óbitos em UTI, de acordo com a população e realidade local.

O perfil dos pacientes admitidos em UTI é dinâmico e, nos últimos anos, foram percebidas alterações epidemiológicas da população e aumento da demanda por leito (FERRETTI-REBUSTINI REL, et al., 2019). Tendo isso em vista, é importante a constante atualização desses dados para auxiliar na elaboração de ações que melhorem o cuidado de pacientes gravemente enfermos e, consequentemente, na redução da morbimortalidade. Portanto, o objetivo deste estudo é caracterizar os perfis social, clínico e hemodinâmico de pacientes admitidos em uma UTI que evoluíram a óbito e verificar se essas variáveis interferem na sobrevida.

\section{MÉTODOS}

\section{Princípios éticos}

Estudo aprovado pelo Comitê de Ética em Pesquisa (CEP) (CAAE 82542017.7.0000.0017), cumprindo os princípios éticos previstos na Resolução 466/12 do Conselho Nacional de Saúde/Conselho Nacional de Ética em Pesquisa. 


\section{Tipo de estudo}

Estudo retrospectivo, descritivo, transversal e inferencial.

\section{População}

Prontuários de pacientes admitidos entre os anos de 2012 a 2018 na UTI de um Hospital de referência que opera em nível terciário na Região Norte do Brasil.

\section{Amostragem e amostra}

A amostra foi de 200 prontuários selecionados por processo de amostragem probabilístico aleatório simples.

\section{Critério de inclusão e exclusão}

Foi utilizado como critério de inclusão a admissão de pacientes na UTI do hospital analisado. Os critérios de exclusão foram: prontuários com dados incompletos ou escassos e pacientes que não foram à UTI.

\section{Desfechos}

Os dados coletados indicaram como características sociais: idade, sexo, raça / cor, profissão, religião, escolaridade e estado civil.

Como características clínicas: diagnóstico principal, estado de gravidade admissional, nível de consciência, grau de mobilidade, suporte ventilatório, frequência de aspirações diárias, presença de sepse, presença de choque, edema, utilização de sondas (nasogástrica, vesical, nasoentérica) e tempo de permanência.

Quanto às características hemodinâmicas: base excess $(\mathrm{BE})$, bicarbonato $\left(\mathrm{HCO}_{3}\right)$, creatinina, hemácias, lactato, leucócitos, plaquetas, potássio potencial de hidrogênio $(\mathrm{pH})$, pressão parcial de gás carbônico $\left(\mathrm{PaCO}_{2}\right)$, sódio e uréia.

Foram considerados como parâmetros de normalidade para a análise das variáveis hemodinâmicas: Base Excess [(-4) - (+2) mEq / L], Bicarbonato [(22 - 26) mEq / L], Creatinina [(0,5 - 1,2 mg) / dL], Hemácias [(3,5 $5,9)$ n. / milhão], Lactato [(<2) mmol/L], Leucócitos [(4.500 - 11.000) n. / milhão], Plaquetas [(150.000 400.000) n. / milhão], Potássio [(3,6 - 5,5) mEq / L], Potencial de Hidrogênio [(7,35 - 7,45)], Pressão parcial de gás carbônico [(35 - 45) mmHg], Sódio [(135 - 145) mEq / L] e Ureia [(8 - 20) mg / dL] (CASTRO D e KEENAGHAN M, 2020; SHAHBAZ H e GUPTA M, 2020; DEAN L, 2005; SHRIMANKER I e BHATTARAI S, 2020; FOUCHER CD e TUBBEN RE, 2020; BARMORE W, et al., 2020).

\section{Análise Estatística}

Os dados foram submetidos à estatística descritiva. As variáveis categóricas foram analisadas pela estatística descritiva, dada pela frequência absoluta e relativa. As variáveis quantitativas foram expressas por média e desvio padrão, após verificar a normalidade dos dados pelo teste Kolmogorov-Smirnov. Além disso, os testes Log-rank, Breslow e Tarone-Ware test foram utilizados para analisar as variáveis independentes ao longo da curva de sobrevida de Kaplan-Meier. As variáveis significativas foram analisadas por Cox Regression para estimar a proporção do evento no período de observação por análise de Exp (B) e pelo intervalo de confiança de 95\% (IC 95\%).

\section{RESULTADOS}

De acordo com as análises, verificamos que o perfil social observado nos pacientes admitidos em terapia intensiva corresponde a indivíduos com a faixa etária entre (20-59 anos) com maior número de pessoas do sexo masculino. Com relação à raça/cor os dados mostram um maior número de pardos. Para as profissões observamos uma diversidade nas atividades laborais apresentadas, no entanto, existe um percentual maior de profissionais declarados como empregada doméstica. Considerando as religiões analisadas observou-se um maior número de católicos. Ainda para o nível educacional o período de escolaridade representativo entre os indivíduos admitidos condiz ao período correspondente a nove anos de escolaridade. Finalmente, os dados relacionados ao estado civil na admissão correspondem aos autodeclarados como solteiro. 
Tabela 1 - Variáveis sociais dos pacientes admitidos em terapia intensiva que evoluíram a óbito, $n=200$. Belém PA, 2020.

\begin{tabular}{|c|c|c|}
\hline Variáveis & $\mathbf{N}$ & $\%$ \\
\hline \multicolumn{3}{|l|}{ Idade } \\
\hline$<19$ anos & 20 & 10 \\
\hline 20 a 59 anos & 97 & 48,5 \\
\hline$\geq 60$ anos & 82 & 41 \\
\hline Não informado & 1 & 0,5 \\
\hline \multicolumn{3}{|l|}{ Sexo } \\
\hline Feminino & 96 & 48 \\
\hline Masculino & 104 & 52 \\
\hline \multicolumn{3}{|l|}{ Raça / cor } \\
\hline Branco & 34 & 17 \\
\hline Pardo & 145 & 72,5 \\
\hline Preto & 9 & 4,5 \\
\hline Não informado & 12 & 6 \\
\hline \multicolumn{3}{|l|}{ Profissão } \\
\hline Agricultor & 5 & 2,5 \\
\hline Aposentado & 11 & 5,5 \\
\hline Autônomo & 5 & 2,5 \\
\hline Do lar & 14 & 7 \\
\hline Empregada doméstica & 21 & 10,5 \\
\hline Estudante & 10 & 5 \\
\hline Lavrador & 8 & 4 \\
\hline Outros & 74 & 37 \\
\hline Não informado & 52 & 26 \\
\hline \multicolumn{3}{|l|}{ Religião } \\
\hline Adventista & 2 & 1 \\
\hline Católico & 11 & 6,5 \\
\hline Evangélico & 9 & 4,5 \\
\hline Outros & 4 & 2 \\
\hline Não informada & 174 & 87 \\
\hline \multicolumn{3}{|l|}{ Nível de escolaridade } \\
\hline Sem escolaridade & 18 & 9 \\
\hline$<09$ anos & 40 & 20 \\
\hline 09 anos & 42 & 21 \\
\hline 12 anos & 26 & 13 \\
\hline$>12$ anos & 13 & 6,5 \\
\hline Não informado & 61 & 30,5 \\
\hline \multicolumn{3}{|l|}{ Estado civil } \\
\hline Casado & 21 & 10,5 \\
\hline Divorciado & 2 & 1 \\
\hline Solteiro & 60 & 30 \\
\hline União estável & 49 & 24,5 \\
\hline Viúvo & 18 & 9 \\
\hline Não informado & 50 & 25 \\
\hline
\end{tabular}

Fonte: Melo GS, et al., 2020.

Observou-se que as principais características clínicas dos indivíduos admitidos incluem: doenças respiratórias, câncer, doenças neurológicas, internação pós-operatórias síndrome da imunodeficiência adquirida e dois ou três diagnósticos associados. Com relação ao estado de gravidade admissional correspondente a pacientes comprometidos, graves, gravíssimos e regulares, houve um maior número de indivíduos com estado grave. Para o nível de consciência categorizado como indivíduos agitados, em coma não induzido, conscientes e desorientados, conscientes e orientados, desorientados e torporosos, os dados 
mostraram um maior número de indivíduos correspondente ao quadro de coma induzido e estado acamado. Ainda com relação ao quadro clínico, um percentual relativo de indivíduos (91\% dos pacientes) necessitava do uso de ventilação mecânica invasiva e a realização de aspirações diárias em $69,5 \%$ dos indivíduos. 0 número de aspiração média foi de 1,275 $\pm 0,549$ por dia. Com relação às complicações clínicas, foram observadas a presença de sepse $(79,5 \%)$, choque $(69 \%)$ e edema $(61 \%)$. Os tipos de sondas necessários no manejo clínico destes pacientes foram nasogástrica $(66,5 \%)$, vesical $(82,5 \%)$, nasoentérica $(36 \%)$. 0 tempo médio de vida foi $11,815 \pm 9,501$ (Tabela 2).

Tabela 2 - Variáveis clínicas dos pacientes admitidos em terapia intensiva que evoluíram a óbito, n=200. Belém PA, 2020.

\begin{tabular}{|c|c|c|}
\hline Variáveis & $\mathbf{N}$ & $\%$ \\
\hline \multicolumn{3}{|l|}{ Diagnóstico principal } \\
\hline Câncer & 36 & 18 \\
\hline Doenças neurológicas & 9 & 4,5 \\
\hline Doenças respiratórias & 41 & 20,5 \\
\hline Pós-operatório & 5 & 2,5 \\
\hline Síndrome da Imunodeficiência Adquirida & 7 & 3,5 \\
\hline Outros & 42 & 21 \\
\hline Dois ou três diagnósticos associados & 60 & 30 \\
\hline \multicolumn{3}{|l|}{ Estado de gravidade } \\
\hline Comprometido & 2 & 1 \\
\hline Grave & 141 & 70,5 \\
\hline Gravíssimo & 15 & 7,5 \\
\hline Regular & 27 & 13,5 \\
\hline Não informado & 15 & 7,5 \\
\hline \multicolumn{3}{|l|}{ Nível de consciência } \\
\hline Agitado & 2 & 1 \\
\hline Coma não-induzido & 3 & 1,5 \\
\hline Coma induzido & 84 & 42 \\
\hline Consciente e desorientado & 2 & 1 \\
\hline Consciente e orientado & 82 & 41 \\
\hline Desorientado & 11 & 5,5 \\
\hline Torporoso & 9 & 4,5 \\
\hline Não informado & 7 & 3,5 \\
\hline \multicolumn{3}{|l|}{ Mobilidade } \\
\hline Acamado & 174 & 87 \\
\hline Deambulante & 6 & 3 \\
\hline Não informado & 20 & 10 \\
\hline \multicolumn{3}{|l|}{ Suporte Ventilatório } \\
\hline Oxigenioterapia & 89 & 44,5 \\
\hline Ventilação mecânica invasiva & 182 & 91 \\
\hline Sepse & 159 & 79,5 \\
\hline Choque & 138 & 69 \\
\hline Edema & 122 & 61 \\
\hline \multicolumn{3}{|l|}{ Sondas } \\
\hline Nasoentérica & 72 & 36 \\
\hline Nasogástrica & 133 & 66,5 \\
\hline Vesical & 165 & 82,5 \\
\hline Aspiração para higiene brônquica & 139 & 69,5 \\
\hline
\end{tabular}

Fonte: Melo GS, et al., 2020.

As variáveis hemodinâmicas estão apresentadas na Tabela 3. Observou-se aumento no valor absoluto das seguintes variáveis: creatinina, uréia, lactato e leucócitos. Os demais parâmetros mostraram-se dentro do padrão de normalidade. 
Tabela 3 - Variáveis hemodinâmicas no momento da admissão em terapia intensiva dos pacientes que evoluíram a óbito. Belém PA, 2020.

\begin{tabular}{ccc}
\hline Variáveis & Média \pm Desvio Padrão & Normalidade \\
\hline Base excess $(\mathrm{BE})(\mathrm{mEq} / \mathrm{L})$ & $1,205 \pm 11,059$ & $(-4)-(+2)$ \\
Bicarbonato $\left(\mathrm{HCO}_{3}\right)(\mathrm{mEq} / \mathrm{L})$ & $20,588 \pm 7,975$ & $22-26$ \\
Creatinina $(\mathrm{mg} / \mathrm{dL})$ & $1,565 \pm 1,714$ & $0,5-1,2$ \\
Hemácias $(\mathrm{n}$ / / 1.000.000) & $3,642 \pm 1,223$ & $3,5-5,9$ \\
Lactato $(\mathrm{mmol} / \mathrm{L})$ & $2,332 \pm 1,595$ & $<2$ \\
Leucócitos $(\mathrm{n} . / 1.000 .000)$ & $16.860 \pm 15.491,02$ & $4.500-11.000$ \\
Plaquetas $(\mathrm{n} . / 1.000 .000)$ & $222.708 \pm 169.206,2$ & $150.000-400.000$ \\
Potássio $(\mathrm{mEq} / \mathrm{L})$ & $3,960 \pm 1,053$ & $3,6-5,5$ \\
Potencial de Hidrogênio $(\mathrm{pH})$ & $7,359 \pm 0,130$ & $7,35-7,45$ \\
Pressão parcial de gás carbônico $\left(\mathrm{PaCO}_{2}\right)\left(\mathrm{mmHg}^{\mathrm{m}}\right)$ & $36,459 \pm 13,209$ & $35-45$ \\
Sódio $(\mathrm{mEq} / \mathrm{L})$ & $140,107 \pm 8,399$ & $135-145$ \\
Ureia $(\mathrm{mg} / \mathrm{dL})$ & $73,837 \pm 70,408$ & $08-20$ \\
\hline
\end{tabular}

Fonte: Melo GS, et al., 2020.

A análise da sobrevida está apresentada na Figura 1. Indivíduos de raça / cor Preta tiveram maior sobrevida que os Brancos na parte central da curva de sobrevida. Além disso, a acentuação dos níveis de leucócitos foi associada com a mortalidade precoce no meio e no final da curva de sobrevida.

Figura 1 - Curva de sobrevida de Kaplan-Meier pacientes admitidos em terapia intensiva, conforme raça e leucócitos.
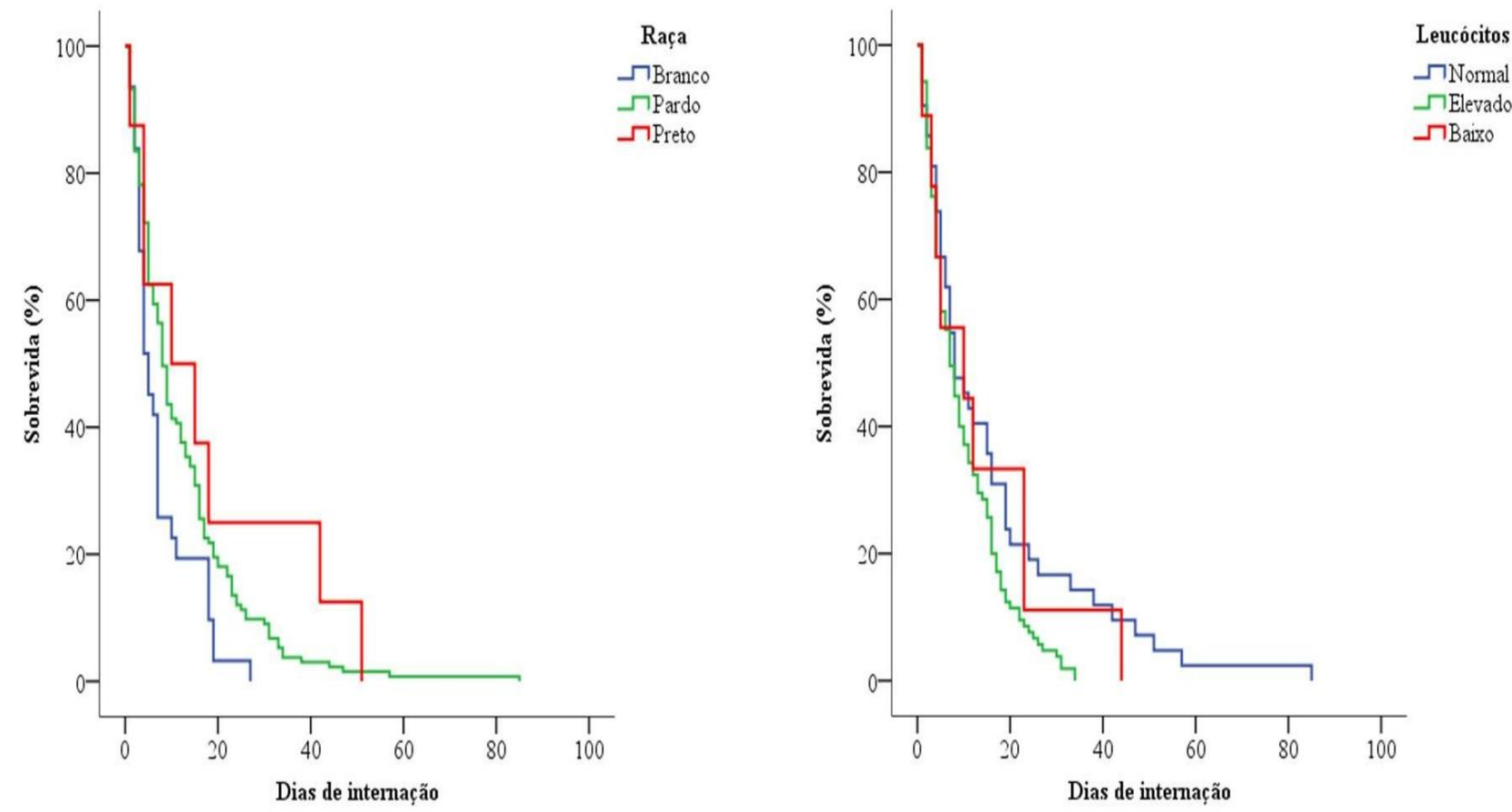

\begin{tabular}{|l|c|c|c|}
\hline & Qui-quadrado df & Sig. \\
\hline Log Rank & 7,703 & 2 & 0,021 \\
\hline Breslow & 5,389 & 2 & 0,068 \\
\hline Tarone-Ware & 6,508 & 2 & 0,039 \\
\hline
\end{tabular}

IC $95 \%$ para $\operatorname{Exp}(\mathbf{B})$ Wald df Sig. $\operatorname{Exp(B)}$ Inferior Superior \begin{tabular}{|l|l|l|l|l|l|r|}
\hline Preto versus Branco & 4,312 & 1 & 0,038 & 2,334 & 1,049 & 5,195 \\
\hline
\end{tabular} \begin{tabular}{|l|l|l|l|l|l|l|}
\hline Preto versus Pardo & 1,006 & 1 & 0,316 & 1,452 & 0,701 & 3,007 \\
\hline
\end{tabular} \begin{tabular}{|l|l|l|l|l|l|}
\hline Branco versus Pardo 5,449 & 1 & 0,02 & 0,622 & 0,417 & 0,927 \\
\hline
\end{tabular}

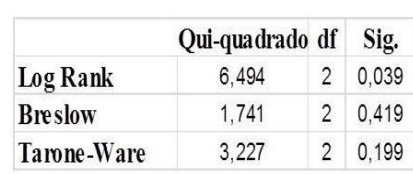

\begin{tabular}{|l|c|c|c|c|c|c|}
\hline & & & & \multicolumn{3}{|c|}{ IC 95\% para Exp(B) } \\
\hline Wormal versus Baixo & 0 & 1 & 0,705 & 1 & 0 & 1,799 \\
\hline Elevado versus Baixo & 1 & 1 & 0,355 & 1,384 & 0,695 & 2,754 \\
\hline Elevado versus Normal & 5,600 & 1 & 0,018 & 1,593 & 1,083 & 2,342 \\
\hline
\end{tabular}

Fonte: Melo GS, et al., 2020. 


\section{DISCUSSÃO}

A internação em terapia intensiva visa reduzir a taxa de óbito relacionada a doenças graves (COOK D e ROCKER G, 2014). O status econômico de uma determinada região pode influenciar em dados epidemiológicos e nas características fisiopatológicas das diferentes doenças (MELO FAF, et al., 2020). Sendo assim, esse estudo objetiva caracterizar o perfil de pacientes admitidos em UTI e verificar se essas variáveis interferem na sobrevida. Os resultados demonstraram as principais variáveis sociais, clínicas e hemodinâmicas destes indivíduos.

Segundo Rodriguez AH, et al. (2016) a quantidade mais expressiva das internações em UTI (19\%) é referente à faixa etária de 50 a 59 anos, seguido da faixa etária de 60 a 69 anos e em terceiro lugar pela faixa etária de 40 a 49 anos. Quando somadas as faixas etárias mais frequentes contata-se que mais de $50 \%$ das internações foram de pacientes com idade entre 40 a 49 anos. Essas faixas etárias se assemelham aos resultados de prevalência deste estudo que aponta a faixa etária de 20 a 59 anos (BONFADA D, et al., 2017). De acordo com estudos de Caldeira VMH, et al. (2010) e Gomes FSL, et al. (2011), o envelhecimento populacional amplia a ocorrência de paciente mais velhos que desenvolvem agravos à saúde, como doenças crônicas e degenerativas, fazendo com que exista uma necessidade maior de tratamento na UTI.

O sexo masculino foi o mais prevalente entre os pacientes admitidos na UTI, representando $52 \%$ do total. Esse resultado é semelhante ao de Rodriguez $\mathrm{AH}$, et al. (2016) em que a prevalência de pacientes do sexo masculino apontou mais da metade com valor de $61,6 \%$, porcentagem que pode ser explicada pela falta de cuidado com a própria saúde pelo gênero masculino, que comumente só adere a medidas governamentais de prevenção à saúde tardiamente, quando a seriedade da enfermidade já está determinada (RODRIGUEZ $\mathrm{AH}$, et al., 2016). O estudo de Oud L (2018) trouxe um resultado similar ao analisar a internação na UTI da população desabrigada, no qual mostrou que $74 \%$ dos pacientes eram homens.

A respeito dos aspectos da raça/cor, este estudo aponta maior frequência de pardos, bem como encontrado por Nascimento MSM, et al. (2018), que caracteriza a população geral da UTI evidenciando que mais da metade da população apresentava essa raça. McNicholas BA, et al. (2018) aborda em sua pesquisa que a raça é um fator demográfico que pode influenciar no tratamento de patologias, como por exemplo, afroamericanos dos quais possuem maior incidência de admissão na UTI com comorbidades secundárias, que incluem diabetes e infecção pelo Vírus da Imunodeficiência Humana, apresentando associação com a sobrevida.

Foi observado neste estudo o catolicismo como religião comum entre os pacientes internados. Freitas KS, et al. (2007) obteve a mesma conclusão e relacionou a religiosidade como um fator social presente e importante em uma UTI, favorecendo a segurança e o bem estar em situações críticas. Seu estudo mostrou que pacientes do setor público e privado gostariam de ser informados sobre o oferecimento de serviços religiosos pelo hospital, o que demonstra a importância de analisar esse dado a fim de representar um recurso interno alternativo para lidar com as questões físicas e mentais de pacientes e familiares, como o agravo da doença e a evolução ao óbito.

O nível de escolaridade no presente estudo foi de nove anos, equivalente ao ensino fundamental completo. Achado semelhante foi encontrado no estudo de El-Fakhouri S, et al. (2016), no qual $63,30 \%$ dos 2.022 pacientes tinham apenas o ensino fundamental completo. Além disso, um estudo realizado com pacientes sépticos na UTI revelou que indivíduos com baixa escolaridade tiveram maior chance de mortalidade e readmissão em 180 dias (SCHNEGELSBERG A, et al., 2016).

O estado civil pode estar associado a outras variáveis como idade e as comorbidades existentes, porém existem estudos que indicam que sua análise independente pode ser preditiva para a associação com a mortalidade hospitalar. Este estudo revelou que pacientes solteiros evoluíram a óbito com mais frequência, assim como no estudo de Seymour CW, et al. (2010) e Metersky ML, et al. (2012) que além desse fator, relacionou o estado civil solteiro a um maior tempo de permanência hospitalar e maior taxa de readmissão, condições que podem ser associados a um menor autocuidado e apoio familiar, mas também a outras questões, como a gravidade das patologias. 
Neste estudo foi encontrado que o estado admissional grave foi o mais comum e esses pacientes apresentam necessidades diferentes (PAPADIMOS TJ, et al., 2011). Outra característica relevante foi a ocorrência coma induzido na admissão. Aslaner MA, et al. (2015) indica que a indução de coma é uma alternativa para pacientes agitados e pacientes graves podem necessitar de sedação e isso, por sua vez, leva a outro aspecto do presente estudo, que é o grau de mobilidade acamado sendo influenciado pela gravidade e pela sedação do coma induzido. Bonfada D, et al (2017) associou o estado de acamados na admissão como um dos preditores de menor taxa de sobrevida em idosos internados na UTI.

Nesse estudo as principais causas patológicas na admissão foram referentes às doenças que acometem o sistema respiratório. El-Fakhouri S, et al. (2016) verificou as principais patologias na admissão, e as doenças do aparelho circulatório foram as mais prevalentes $(25,5 \%)$, e do aparelho respiratório foi apenas $9 \%$.

O número de pacientes que necessitou de ventilação mecânica invasiva (VMI) foi elevado no presente estudo. Fialkow L, et al. (2016) também teve alta porcentagem de pacientes que utilizaram VMI, cerca de $97,7 \%$, e os principais motivos para o início da VMI foram sepse, choque e pneumonia. E o modo de ventilação sob pressão assistida / controlada foi o mais utilizado.

A maioria dos indivíduos internados precisou de aspirações diárias, a aspiração é importante para a higiene bucal e prevenção de pneumonia associada ao ventilador, que é uma das principais causas de complicações durantes a internação, que leva ao aumento do risco de mortalidade e de maior tempo de permanência na UTI. A intubação traqueal e a imobilidade pioram a efetividade da tosse e do movimento ciliar, levando ao acúmulo de secreções, por isso é importante a avaliação da necessidade de aspiração diariamente (HUA F, et al., 2016; ASSMANN CB, 2016).

No presente estudo, uma numeração expressiva dos pacientes que foram a óbito desenvolveram sepse durante a internação $(79,5 \%)$. Em um estudo realizado em um hospital universitário no Brasil, mostrou que cerca de $31 \%$ dos pacientes tiveram sepse como complicação durante a internação, e destes $39 \%$ foram a óbito. $E$ os pacientes que tiveram sepse $52 \%$ desenvolveram choque séptico, o que está relacionado a um elevado risco de mortalidade (MORELLO LG, et al., 2019).

O tempo de internação desses pacientes na UTI foi em média de 11 dias. Rodriguez AH, et al. (2016), mostra que o tempo médio de internação na UTI foi de 6 dias, e a maioria dos pacientes ficaram internados de 0 a 3 dias (53,2\%) e apenas $9,1 \%$ permaneceram por 11 a 15 dias. Conhecer o tempo médio de internação é importante para que os profissionais possam planejar as intervenções preventivas e utilizar da melhor forma os recursos hospitalares, e serve como parâmetro para análise da gravidade das doenças (AWAD A, et al., 2017).

Neste estudo, observou-se que os pacientes que foram a óbito apresentaram níveis elevados de lactato. Estudos prévios de Bou Chebl R, et al. (2017) e Villar J, et al. (2019) a respeito da associação dos níveis aumentados de lactato com a mortalidade (curto e longo prazo), discorrem que a hipoperfusão tecidual resulta em estresse oxidativo e metabólico, o que pode levar a muitas condições como a sepse, o choque, a insuficiência múltipla de órgãos, entre outras ocorrências. O que mostra a independência dessa variável e a heterogeneidade da população de UTI (diferentes patologias e acometimentos).

A função renal é principalmente analisada por meio dos níveis séricos de creatinina e ureia. Estes compostos também estão relacionados com a situação nutricional. No presente estudo, os níveis apresentaram aumento em pacientes internados em UTI que evoluíram a óbito no momento da admissão. Pacientes sob cuidados intensivos apresentam a função renal sobrecarregada, logo, apresentam filtração glomerular reduzida, desequilíbrios acidobásicos e hidroeletrolíticos, gerando acúmulo de substâncias e evoluir à lesão renal aguda, a qual se mostrou associada à mortalidade em UTI nos estudos (PERES LAB, et al., 2015; SAXENA A e MESHRAM SV , 2018).

A ocorrência de leucocitose é frequente em decorrência de o paciente crítico passar por processos inflamatórios acentuado, infecções (por diversos agentes como bactérias, vírus e fungos), uso de certos medicamentos e outros fatores (ASADOLLAHI K, et al., 2011; GUTIERREZ-RODRIGUEZ R, et al., 2015). Neste sentido, estudos prévios de Asadollahi K (2011) e Gutierrez-Rodriguez R (2015) evidenciam a 
leucocitose como fator preditivo à mortalidade em pacientes sob cuidados intensivos, assim como os resultados desta pesquisa. Além disso, observou-se que estes apresentaram também apresentam uma menor sobrevida.

As limitações para este trabalho incluíram o processo da coleta dos dados, no que diz respeito à localização e ao acesso imediato aos prontuários, bem como dados incompletos de algumas variáveis. Estas dificuldades são pertinentes aos estudos epidemiológicos, principalmente realizados no Brasil (TIMOTEO F, et al., 2020).

\section{CONCLUSÃO}

Concluímos que as principais características sociais observadas nos pacientes que evoluíram a óbito neste estudo foram da faixa etária entre 20 a 59 anos, sexo masculino, raça/cor Pardo, empregadas domésticas, católicos, escolaridade de 9 anos e estado civil solteiros. Quanto aos aspectos clínicos, os mais frequentes foram presença de doenças respiratórias, estado grave na admissão, coma induzido, acamados, sendo necessário o uso de ventilação mecânica invasiva e a realização de aspirações diárias na maioria dos indivíduos internados. Em relação às variáveis hemodinâmicas, os parâmetros de creatinina, uréia, lactato e leucócitos sofreram aumento. Além disso, foi observado que indivíduos que apresentaram leucocitose e de raça/cor Branca apresentam menor sobrevida. Portanto, este estudo auxilia na compreensão do perfil epidemiológico da população local, contribuindo no melhor manejo do paciente crítico.

\section{REFERÊNCIAS}

1. ABE T, et al. Characteristics, management, and in-hospital mortality among patients with severe sepsis in intensive care units in Japan: the FORECAST study. Critical Care, 2018; 22(1): 322 .

2. ASADOLLAHI K, et al. Leukocytosis as an alarming sign for mortality in patients hospitalized in general wards. Iranian Journal of Medical Sciences, $2011 ; 36(1): 45-49$.

3. ASLANER MA, et al..Admissions of Critically III Patients to the ED Intensive Care Unit. American Journal of Emergency Medicine, 2015; 33(4):501-5.

4. ASSMANN CB. Hiperinsuflação pulmonar com ventilador mecânico versus aspiração traqueal isolada na higiene brônquica de pacientes submetidos à ventilação mecânica. Revista Brasileira de Terapia Intensiva, 2016; 28(1): 2732.

5. AWAD A, et al. Patient Length of Stay and Mortality Prediction: A Survey. Health Services Management Research, 2017; 30(2): 105-120.

6. BARMORE W, et al., 2020. Physiology, Urea Cycle. In: StatPearls. Treasure Island (FL): StatPearls Publishing. Disponível em: https://www.ncbi.nlm.nih.gov/books/NBK513323/. Acesso em: 17 mai. 2020.

7. BONFADA D, et al. Survival analysis of elderly patients in Intensive Care Units. Revista Brasileira de Geriatria e Gerontologia, 2017; 20(2), 197-205.

8. BOU CHEBL $R$, et al. Serum lactate is an independent predictor of hospital mortality in critically ill patients in the emergency department: a retrospective study. Scandinavian Journal of Trauma, Resuscitation and Emergency, 2017; 25(1): 69.

9. CALDEIRA VMH, et al. Critérios para admissão de pacientes na unidade de terapia intensiva e mortalidade. Revista da Associação Médica Brasileira, 2010; 56(5): 528-534.

10. CANO AG, et al. Evidence on the utility of hemodynamic monitorization in the critical patient. Medicina Intensiva, 2012; 36(9): 650-655.

11. CASTRO D, KEENAGHAN M, 2020. Arterial Blood Gas. In: StatPearls. Treasure Island (FL): StatPearls Publishing. Disponível em: https://www.ncbi.nlm.nih.gov/books/NBK536919/. Acesso em: 17 mai. 2020.

12. COOK D, ROCKER G, Dying with Dignity in the Intensive Care Unit. The New England Journal of Medicine, 2014; 370 : 2506-2514.

13. DEAN L, 2005. Blood Groups and Red Cell Antigens. Bethesda (MD): National Center for Biotechnology Information (US), Table 1, Complete blood count. Disponível em: https://www.ncbi.nlm.nih.gov/books/NBK2263/table/ch1.T1/. Acesso em: 17 mai. 2020.

14. EL-FAKHOURI S, et al. Perfil epidemiológico dos pacientes da UTI da Faculdade de Medicina de Marília. Revista da Associação Médica Brasileira, 2016; 62(3): 248-254.

15. FERRETTI-REBUSTINI REL, et al. Level of acuity, severity and intensity of care of adults and older adults admitted to the Intensive Care Unit. Revista da Escola de Enfermagem da USP, 2019; 53: e03416.

16. FIALKOW L, et al. Ventilação mecânica em pacientes da unidade de terapia intensiva de um hospital universitário geral do sul do Brasil: um estudo epidemiológico. Clinics, 2016; 71(3), 144-151.

17. FOUCHER CD, TUBBEN RE, 2020. Lactic Acidosis. In: StatPearls [Internet]. Treasure Island (FL): StatPearls Publishing. Disponível em: https://www.ncbi.nlm.nih.gov/books/NBK470202/. Acesso em: 17 mai. 2020. 
18. FREITAS KS. Family members' needs at intensive care units: comparative analysis between a public and a private hospital. Revista Latino-Americana de Enfermagem, 2007; 15(1): 84-92.

19. GOMES FSL, et al. Avaliação de risco para úlcera por pressão em pacientes críticos. Revista da Escola de Enfermagem da USP, 2011; 45(2): 313-318.

20. GUTIERREZ-RODRIGUEZ R, et al. Lymphopenia assessment in ICU patients and relationship with mortality. Intensive Care Medicine Experimental, 2015; 3(Suppl 1): A340.

21. HUA F, et al. Oral Hygiene Care for Critically III Patients to Prevent Ventilator-Associated Pneumonia. The Cochrane Database of Systematic Reviews, 2016; 10(10):CD008367.

22. McNICHOLAS BA, et al. Lessons to learn from epidemiologic studies in ARDS. Current Opinion in Critical Care. 2018; 24(1): 41-48.

23. MELO FAF, et al. A systematic review and meta-analysis of acute kidney injury in the intensive care units of developed and developing countries.PLoS One, 2020; 15(1): e0226325.

24. METERSKY ML, et al. The effect of marital status on the presentation and outcomes of elderly male veterans hospitalized for pneumonia. Chest, 2012; 142(4): 982-987.

25. MINISTÉRIO DA SAÚDE. Portaria $\mathrm{n}^{\circ}$ 895, de 31 de março de 2017. Disponível: http://portalarquivos.saude.gov.br/images/pdf/2017/abril/07/106713-16-82-Minuta-Portaria-PROTOCOLO.pdf. Acesso em: 22 abr. 2020

26. MORELLO LG, et al. Avaliação das características clínicas e epidemiológicas de pacientes com e sem sepse em unidades de terapia intensiva de um hospital terciário. Einstein (São Paulo), 2019; 17 (2): eAO4476.

27. NASCIMENTO MSM, et al. Perfil epidemiológico de pacientes em unidade de terapia intensiva adulto de um hospital regional paraibano. Temas em saúde, 2018; 18(1): 247-265.

28. OUD L. Epidemiology, Characteristics, and Outcomes of ICU-Managed Homeless Patients: A Population-Based Study. BioMed Research International. 2018; 1-11.

29. PAPADIMOS TJ, et al. An overview of end-of-life issues in the intensive care unit. International Journal of Critical Illness and Injury Science, 2011; 1(2): 138-146.

30. PERES LAB, et al. Predictors of acute kidney injury and mortality in an Intensive Care Unit. Brazilian Journal of Nephrology, 2015; 37(1): 38-46.

31. RODRIGUEZ AH, et al. Características epidemiológicas e causas de óbitos em pacientes internados em terapia intensiva. Revista Brasileira de Enfermagem, 2016; 69(2), 229-234.

32. SAXENA A, MESHRAM SV. Predictors of Mortality in Acute Kidney Injury Patients Admitted to Medicine Intensive Care Unit in a Rural Tertiary Care Hospital. Indian Journal of Critical Care Medicine. 2018; 22(4): 231-237.

33. SCHNEGELSBERG A, et al. Impact of Socioeconomic Status on Mortality and Unplanned Readmission in Septic Intensive Care Unit Patients. Acta anaesthesiologica Scandinavica, 2016; 60(4): 465-75.

34. SEYMOUR CW, et al. Marital Status and the Epidemiology and Outcomes of Sepsis. Chest, 2010; 137(6): 1289-1296.

35. SHAHBAZ H, GUPTA M, 2020 Creatinine Clearance. In: StatPearls [Internet]. Treasure Island (FL): StatPearls Publishing. Disponível em: https://www.ncbi.nlm.nih.gov/books/NBK544228/. Acesso em: 17 mai. 2020.

36. SHRIMANKER I, BHATTARAI S, 2020. Electrolytes. In: StatPearls. Treasure Island (FL): StatPearls Publishing. Disponível em: https://www.ncbi.nlm.nih.gov/books/NBK541123/. Acesso em: 17 mai. 2020.

37. TIMOTEO F, et al. Bladder cancer trends and mortality in the brazilian public health system, International Brazilian Journal of Urology, 2020; 46(2): 224-233.

38. VILLAR J, et al. Lactate Predicts Both Short- and Long-Term Mortality in Patients With and Without Sepsis. Infectious Diseases, 2019; 12: 1178633719862776.

39. XIA DL, et al. Cell-free DNA increase over first 48 hours in emergency intensive care unit predicts fatal outcome in patients with shock. Journal of International Medical Research, 2016; 44(5): 1002-1012. 